The benefits of the touch screen system in this trial may have been underestimated by contamination and high baseline levels of knowledge. Graham and colleagues rightly state that: "Like all new technologies, these devices should be subject to rigorous evaluation." With limited evidence of benefit for these expensive tools over well designed leaflets, they seem to fit best into the National Institute for Clinical Excellence (NICE) category C: for NHS use only in the context of rigorous research studies. ${ }^{7}$
1 Smith R. The future of healthcare systems: information technology and consumerism will transform health care world wide. BMJ 1997;314:1495-6.

2 Wyatt JC. Same information, different decisions: format counts. BMJ 1999:318:1501-2.

3 Altman DG, Bland JM. Units of analysis. BMJ 1998;314:1874.

4 Schultz KF, Chalmers I, Hayes RJ, Altman DG. Dimensions of methodological quality associated with estimates of treatment effects in controlled trials. JAMA 1995;273:408-12.

5 Streiner DL, Norman GR. Health measurement scales. Oxford: Oxford University Press, 1995:80

6 Peto R, Collins R, Gray R. Large-scale randomised evidence: large, simple trials and overviews of trials. Ann N Y Acad Sci 1993;703:314-40.

7 Rawlins M. In pursuit of quality: the National Institute for Clinical Excellence. Lancet 1999;353:1079-82.

\title{
INFOPOINTS
}

\section{Managing information overload: developing an electronic directory}

General practitioners need convenient access to a wide range of accurate information to support clinical practice. ${ }^{1}$ The sheer volume of such information works against doctors quickly locating the information they want. ${ }^{2}$ This problem has stimulated interest in electronic methods of organising and accessing information. ${ }^{3}$

With this in mind, we developed a centralised information service and electronic directory of healthcare services for general practitioners in the Brighton, Hove, and Lewes areas of East Sussex. We constructed the directory using WAX Active Library software (www.medinfo.cam.ac.uk/wax), which was designed specifically for use in primary care. We asked all the major healthcare trusts, service providers, and community and social services organisations in the region to provide details of their services, contact information, and relevant clinical guidelines, policies, and referral procedures. We used existing directories where possible, but the directory was compiled predominantly from scraps of publicly available information and supplemented with new information written for the purpose. Very little of the directory content was provided electronically, which necessitated resource-intensive manual scanning of documents and text conversion before they could be added to the directory.

Despite perceptions of an abundance of information, most available information was of poor quality or not in a format that allowed for easy use by general practitioners. Other problems we faced during the directory's development included

- Little awareness among health agencies of the importance of good quality information

- Little appreciation among trusts of the value of promoting their services and referral procedures to general practitioners

- Some reticence towards openly sharing information, often expressed as a fear of potential misuse

- Information related to healthcare services was largely non-existent

- Generally poor computerisation in general practices.

The pilot study involved installing the directory on 66 personal computers in 10 self selected local surgeries. Thirty $(45 \%)$ of the computers were used solely by general practitioners, who accounted for the highest level of directory use. Average daily use by all users dur- ing the pilot was 2.3 occasions per computer (range 1.2-7.2). The information categories that were most frequently accessed related to hospital trusts, social services departments, voluntary agencies, and local practitioners (comprising 82\% of all content viewed). Use was highest among individuals who received training in the directory's use. Participants were positive about the directory's comprehensiveness, local relevance, simplicity of use, and speed and efficiency in accessing information when needed. In most cases users were able to locate the required information in 15-30 seconds.

After the pilot's success, the directory was made available free of charge to local practices, with quarterly updates (on CD Rom). There are plans to extend the service.

Senior healthcare managers in our region now publicly espouse the benefits of a central information service for primary care. The reasons for this shift are twofold. Firstly, since using the electronic directory, many general practitioners have brought pressure to bear on their local trusts to improve the quality of their information. Secondly, having a demonstrable product, instead of what was once little more than a theoretical vision, means that individuals can now appreciate firsthand its practical applications at the clinical coalface.

These small advances notwithstanding, the need to develop a sustainable information culture in healthcare services cannot be underestimated-particularly if the NHS information strategy is to be realised.

Jennie Lyons, Primary Care Information Service coordinator

Alex Khot, general practitioner

Primary Care Information Service, PO Box 11, Portslade BN41 1XY

Competing interests: None declared.

1 Smith R. What clinical information do doctors need? BMJ 1996;313: 1062-8.

2 Hibble A, Kanka D, Pencheon D, Pooles F. Guidelines in general practice: the new Tower of Babel? BMJ 1998;317:862-3.

3 Fraser HSF, Kohane IS, Long WJ. Using the technology of the world wide web to manage clinical information. BMJ 1997;314:1600-3. 